\title{
Definable Transductions and Weighted Logics for Texts *
}

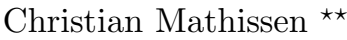 \\ Institut für Informatik, Universität Leipzig \\ D-04009 Leipzig, Germany \\ mathissen@informatik. uni-leipzig.de
}

\begin{abstract}
A text is a word together with an additional linear order on it. We study quantitative models for texts, i. e. text series which assign to texts elements of a semiring. We consider an algebraic notion of recognizability following Reutenauer and Bozapalidis and show that recognizable text series coincide with text series definable in weighted logics as introduced by Droste and Gastin. In order to do so, we study certain definable transductions and show that they are compatible with weighted logics. Moreover, we show that the behavior of weighted parenthesizing automata coincides with certain definable series.
\end{abstract}

\section{Introduction}

Texts as introduced by Rozenberg and Ehrenfeucht 9 ] extend the model of words by an additional linear order. The theory of texts originates in the theory of 2structures (cf. [8]) and it turns out that texts represent an important subclass of 2-structures, namely T-structures [10]. Moreover, Ehrenfeucht and Rozenberg proposed texts as a well-suited model for natural texts that may carry in its tree-like structure grammatical information [10, p.264].

A number of authors [11,14, 15] have investigated classes of text languages such as the families of context-free, equational or recognizable text languages and developed a language theory. In particular, the fundamental result of Büchi on the coincidence of recognizable and definable languages has been extended to texts [15]. Recently, Droste and Gastin [5] introduced weighted logics over words and showed a Büchi-type characterization for weighted automata over words. They enrich the language of monadic second order logic with values from a semiring in order to add quantitative expressiveness. Since they define their logic for arbitrary commutative semirings, the framework is very flexible, e.g. one may now express how often a certain property holds, how much execution time a process needs or how reliable it is. The result of Droste and Gastin has been extended to trees, traces, pictures and infinite words [7, 17, 16, 6].

In this paper we consider quantitative aspects of texts and study weighted logics for them. We extend both results, that of Hoogeboom and ten Pas to a

\footnotetext{
* Appeared in: Proc. of the 11th DLT, Turku, LNCS 4588, pp.324-336, 2007.

** Supported by the GK 446 of the German Research Foundation
} 
weighted setting and that of Droste and Gastin to texts. However, rather than using a combinatorial automaton model we follow Hoogeboom and ten Pas who considered recognizability in the algebraic sense. We regard a weighted algebraic recognizability concept for general algebras following a line of research initiated by Reutenauer [19] and continued by Bozapalidis [2]. It generalizes weighted automata on words and trees as well as the notion of recognizable languages as defined by Mezei and Wright in the 1960s [18].

In order to show the coincidence of recognizable series with the ones definable by certain sentences in weighted logics, we refine the transductions from texts to terms and vice versa given by Hoogeboom and ten Pas such that they are compatible with weighted logics. Therefore, we study a certain subclass of Courcelle's definable transductions [3] and show that it preserves definability with respect to weighted logics. This tool enables us to easily transfer results on weighted logics to different structures.

An important subclass of texts, the class of alternating texts, forms the free bisemigroup and is isomorphic to the class of the so-called sp-biposet introduced by Ésik and Németh in [12. In the last section we will generalize the parenthesizing automata of Ésik and Németh to a weighted setting and show that their behaviors are exactly the series definable by certain sentences in weighted logics.

We point out that our method extends to classes of graphs where there are similar pairs of transductions as for texts. This applies e.g. to classes of graphs where the modular decompositions can be defined by certain restricted formulae in the graph itself, i. e. to classes of graphs that are, in terminology of Courcelle, "RMSO-parsable". This will be subject of further research.

\section{Recognizable Series over General Algebras}

Let $\Sigma$ be a finite ranked alphabet interpreted as a functional signature and let $\operatorname{rk}(f) \in \mathbb{N}$ denote the rank of $f$ for all $f \in \Sigma$. Let $\mathcal{C}$ be a finitely generated $\Sigma$ algebra. We fix a finite generating set $\Delta \subseteq \mathcal{C}$. We recall the following definition:

Definition 2.1 (Mezei \& Wright [18]). A $\mathcal{C}$-language $L \subseteq \mathcal{C}$ is recognizable if there is a finite $\Sigma$-algebra $\mathscr{A}$ and a homomorphism $\varphi: \mathcal{C} \rightarrow \mathscr{A}$ such that $\varphi^{-1}(\varphi(L))=L$.

The free $\Sigma$-algebra over $\Delta$ is denoted $T_{\Sigma}(\Delta)$ and comprises all $\Sigma$-terms or equivalently all $\Sigma$-trees over $\Delta$. Let $\eta_{\mathcal{C}}: T_{\Sigma}(\Delta) \rightarrow \mathcal{C}$ denote the unique epimorphism extending id $(\Delta)$. Let $x$ be a fresh symbol. The set of contexts $\operatorname{CTX}(\Sigma, \Delta) \subseteq T_{\Sigma}(\Delta \cup\{x\})$ is the set of trees where $x$ appears at exactly one leaf. For $s \in \mathcal{C}$ and $\tau \in \operatorname{CTX}(\Sigma, \Delta), \tau[s]$ denotes the value of the term function of $\tau$ on $\mathcal{C}$ at $s$.

Similar to Definition 2.1, we introduce a concept of recognizability for (formal) $\mathcal{C}$-series, i.e. for functions from $\mathcal{C}$ to a semiring $\mathbb{K}$. A semiring $\mathbb{K}$ is an algebraic structure $(\mathbb{K},+, \cdot, 0,1)$ such that $(\mathbb{K},+, 0)$ is a commutative monoid, $(\mathbb{K}, \cdot, 1)$ is a monoid, multiplication distributes over addition and 0 acts absorbing. If multiplication is commutative, then $\mathbb{K}$ is a commutative semiring. If addition is idempotent, then $\mathbb{K}$ is an idempotent semiring. We call a semiring locally 
finite if any finitely generated subsemiring is finite. Examples for semirings comprise the trivial Boolean algebra $\mathbb{B}=(\{0,1\}, \vee, \wedge, 0,1)$ and the natural numbers $(\mathbb{N},+, \cdot, 0,1)$ as well as the tropical semiring $(\mathbb{N} \cup\{\infty\}, \min ,+, \infty, 0)$ and the arctical semiring $(\mathbb{N} \cup\{-\infty\}$, max $,+,-\infty, 0)$ which are used to model problems in operations research. Important examples are also the probabilistic semiring $([0,1], \max , \cdot, 0,1)$ and the semiring of formal languages $\left(\mathscr{P}\left(\Delta^{*}\right), \cup, \cap, \emptyset, \Delta^{*}\right)$. Let in the sequel $\mathbb{K}$ be a commutative semiring such that $0 \neq 1$.

A $\mathbb{K}$-semimodule $M$ is an Abelian monoid $(M,+)$ together with a scalar multiplication $\cdot: \mathbb{K} \times M \rightarrow M$ such that for all $k, l \in \mathbb{K}$ and $m, n \in M$ we have

$$
\begin{aligned}
k \cdot(m+n) & =k \cdot m+k \cdot n, \quad(k+l) \cdot m & =k \cdot m+l \cdot m, \quad(k l) \cdot m=k \cdot(l \cdot m), \\
1 \cdot m & =m, \quad 0 \cdot m & =0 .
\end{aligned}
$$

A submonoid $N$ of $M$ is a subsemimodule if $\mathbb{K} N \subseteq N$. It is finitely generated if $N=\mathbb{K} \cdot m_{1}+\ldots+\mathbb{K} \cdot m_{n}$ for some $m_{1}, \ldots, m_{n} \in M$.

A $\mathbb{K}$ - $\Sigma$-algebra $\mathscr{A}=\left(\mathscr{A},\left(\mu_{f}\right)_{f \in \Sigma}\right)$ consists of a $\mathbb{K}$-semimodule $\mathscr{A}$ together with multilinear operations $\mu_{f}$ of $\operatorname{rank} \operatorname{rk}(f)$ (cf. [1]). Letting $\mu_{f}$ interpret the function symbol $f \in \Sigma, \mathscr{A}$ becomes a $\Sigma$-algebra. A $\mathbb{K}$ - $\Sigma$-algebra is said to have finite rank if it is a finitely generated $\mathbb{K}$-semimodule.

Example 2.2. In the following, $\mathbb{K}\langle\langle\mathcal{C}\rangle\rangle$ denotes the set of (formal) $\mathcal{C}$-series. Together with pointwise addition and the scalar multiplication $(k \cdot S, s)=k \cdot(S, s)$ for all $k \in \mathbb{K}, S \in \mathbb{K}\langle\langle\mathcal{C}\rangle\rangle$ and $s \in \mathcal{C}$ it is a $\mathbb{K}$-semimodule. The set $\mathbb{K}\langle\mathcal{C}\rangle$ of series $P$ having finite support, i. e. where $\{s \in \mathcal{C} \mid(P, s) \neq 0\}$ is finite, is a subsemimodule of $\mathbb{K}\langle\langle\mathcal{C}\rangle\rangle$. It is the free $\mathbb{K}$-semimodule over $\mathcal{C}$. Hence, any $S: \mathcal{C} \rightarrow \mathbb{K}$ extends linearly to $\mathbb{K}\langle\mathcal{C}\rangle$. We will not distinguish between $S$ and its linear extension. $P \in \mathbb{K}\langle\mathcal{C}\rangle$ is called polynomial.

We equip the $\mathbb{K}$-semimodule $\mathbb{K}\langle\mathcal{C}\rangle$ with multilinear operations in order to make it a $\mathbb{K}$ - $\Sigma$-algebra. We define

$$
\left(\mu_{f}\left(P_{1}, \ldots, P_{n}\right), s\right)=\sum_{\substack{s_{1}, \ldots, s_{n} \in \mathcal{C} \\ f\left(s_{1}, \ldots, s_{n}\right)=s}}\left(P_{1}, s_{1}\right) \cdot \ldots \cdot\left(P_{n}, s_{n}\right) .
$$

Note, as the $P_{i}$ are polynomials, the sum is in fact finite. It is not hard to see that this definition indeed gives multilinear operations $\mu_{f}$. Hence, $\mathbb{K}\langle\mathcal{C}\rangle$ is a $\mathbb{K}$ $\Sigma$-algebra and thus a $\Sigma$-algebra. Identifying $s \in \mathcal{C}$ with the polynomial that maps $s$ to 1 and any other element of $\mathcal{C}$ to $0, \mathcal{C}$ becomes a subalgebra of $\mathbb{K}\langle\mathcal{C}\rangle$.

We interpret $\mathbb{K}$ - $\Sigma$-algebras as algebras in the sense of universal algebra over the signature $\left(+,(k \cdot)_{k \in \mathbb{K}},\left(\mu_{f}\right)_{f \in \Sigma}\right)$. Semimodules are algebras over the signature $\left(+,(k \cdot)_{k \in \mathbb{K}}\right)$. The notion of a $\mathbb{K}-\Sigma$-homomorphism and a $\mathbb{K}$ - $\Sigma$-epimorphism as well as the notion of a congruence are defined as usual in universal algebra.

Remark 2.3. It is not hard to see that $\mathbb{K}\left\langle T_{\Sigma}(\Delta)\right\rangle$ is the free $\mathbb{K}$ - $\Sigma$-algebra over $\Delta$. Hence, for any $\mathbb{K}$ - $\Sigma$-algebra $\mathscr{A}$, any mapping $\mu_{\mathscr{A}}: \Delta \rightarrow \mathscr{A}$ extends uniquely to a $\mathbb{K}$ - $\Sigma$-homomorphism $\mu_{\mathscr{A}}: \mathbb{K}\left\langle T_{\Sigma}(\Delta)\right\rangle \rightarrow \mathscr{A}$. 
For any function $h: A \rightarrow B$ the kernel of $h$ denoted $\operatorname{ker}(h)$ is the set $\{(x, y) \in$ $\left.A^{2} \mid h(x)=h(y)\right\}$. If $h$ is a homomorphism, then $\operatorname{ker}(h)$ is a congruence. Now, we are ready to define a general notion of weighted recognizability.

Definition 2.4. A $\mathcal{C}$-series $S: \mathcal{C} \rightarrow \mathbb{K}$ is recognizable if there is a $\mathbb{K}$ - $\Sigma$-algebra of finite rank $\mathscr{A}$ and a $\mathbb{K}-\Sigma$-epimorphism $\varphi: \mathbb{K}\langle\mathcal{C}\rangle \rightarrow \mathscr{A}$ such that $\operatorname{ker}(\varphi) \subseteq$ $\operatorname{ker}(S)$.

Note that the definition is independent of the set of constants, i. e. independent of the symbols of $\Sigma$ of rank 0 . Hence, we may e.g. add constants from $\Delta$ to $\Sigma$ without altering the class of recognizable series.

First, we show that Definition 2.4 generalizes Definition 2.1. For a language $L \subseteq \mathcal{C}$ let $\mathbb{1}_{L}$ denote the characteristic series of $L$.

Proposition 2.5. A language $L \subseteq \mathcal{C}$ is recognizable iff $\mathbb{1}_{L}: \mathcal{C} \rightarrow \mathbb{B}$ is recognizable.

Proof. (If). Let $L \subseteq \mathcal{C}$ and let $\mathbb{1}_{L}: \mathcal{C} \rightarrow \mathbb{B}$ be recognized by $\varphi: \mathbb{B}\langle\mathcal{C}\rangle \rightarrow \mathscr{A}$. Since $\operatorname{ker}(\varphi) \subseteq \operatorname{ker}(S)$, it is easy to see that $\varphi_{\mid \mathcal{C}}$ saturates $L$.

(Only if). Let $L \subseteq \mathcal{C}$ be recognized by $\varphi: \mathcal{C} \rightarrow \mathscr{A}$. We extend $\varphi$ to a $\mathbb{B}$ - $\Sigma$-epimorphism $\varphi: \mathbb{B}\langle\mathcal{C}\rangle \rightarrow \mathbb{B}\langle\varphi(\mathcal{C})\rangle$. We show that $\operatorname{ker}(\varphi) \subseteq \operatorname{ker}(S)$ which concludes the proof. Let $P_{1}, P_{2} \in \mathcal{C}$ with $\varphi\left(P_{1}\right)=\varphi\left(P_{2}\right)$. We may interpret $P_{1}, P_{2}$ as finite subsets of $\mathcal{C}$. We have $\left(S, P_{1}\right)=1$ iff there is $c_{1} \in P_{1}$ with $\left(S, c_{1}\right)=1$ iff there is $c_{1} \in P_{1}$ with $\varphi\left(c_{1}\right) \in \varphi(L)$ iff there is $c_{2} \in P_{2}$ with $\varphi\left(c_{2}\right) \in \varphi(L)$ iff $\left(S, P_{2}\right)=1$. Hence, $\left(S, P_{1}\right)=\left(S, P_{2}\right)$.

We say a formal power series $S: \Delta^{*} \rightarrow \mathbb{K}$ is regular if it is the behavior of some weighted finite automaton. Reutenauer proved the following for commutative rings. His proof also works for locally finite commutative semirings.

Proposition 2.6 (Reutenauer [19]). Let $\mathbb{K}$ be a commutative ring or let $\mathbb{K}$ be a locally finite commutative semiring. A formal power series is recognizable iff it is regular.

Let $S: \mathcal{C} \rightarrow \mathbb{K}$ and let $\sim_{S}=\left\{\left(P_{1}, P_{2}\right) \in \mathbb{K}\langle\mathcal{C}\rangle \times \mathbb{K}\langle\mathcal{C}\rangle \mid\left(S, \tau\left[P_{1}\right]\right)=\right.$ $\left(S, \tau\left[P_{2}\right]\right)$ for all $\left.\tau \in \operatorname{CTX}(\Sigma, \Delta)\right\}$. It is not hard to see that this is a $\mathbb{K}-\Sigma$ congruence. Let $\sim$ be any congruence contained in $\operatorname{ker}(S)$ and let $\left(P_{1}, P_{2}\right) \in \sim$. Then $\left(\tau\left[P_{1}\right], \tau\left[P_{2}\right]\right) \in \sim$ for any $\tau \in \operatorname{CTX}(\Sigma, \Delta)$ as $\sim$ is a congruence. Therefore, we have $\left(S, \tau\left[P_{1}\right]\right)=\left(S, \tau\left[P_{2}\right]\right)$ for all $\tau \in \operatorname{CTX}(\Sigma, \Delta)$. This shows that $\sim \subseteq \sim_{S}$ and, hence, that $\sim_{S}$ is the greatest congruence fully contained in $\operatorname{ker}(S)$. We define $\mathscr{A}_{S}=\mathbb{K}\langle\mathcal{C}\rangle / \sim_{S}$, the syntactic $\mathbb{K}$ - $\Sigma$-algebra of $S$. Note this definition is independent of the choice of $\Delta$. We conclude:

Proposition 2.7. A series $S: \mathcal{C} \rightarrow \mathbb{K}$ is recognizable iff $\mathscr{A}_{S}$ is of finite rank.

Lemma 2.8. Let $\mathcal{C}_{1}, \mathcal{C}_{2}$ be finitely generated $\Sigma$-algebras, let $\psi: \mathcal{C}_{1} \rightarrow \mathcal{C}_{2}$ be an epimorphism and let $S: \mathcal{C}_{2} \rightarrow \mathbb{K}$. Then $\psi^{-1}(S)$ is recognizable iff $S$ is recognizable. 
Proof. (If). Extend $\psi$ linearly to a $\mathbb{K}$ - $\Sigma$-epimorphism $\psi: \mathbb{K}\left\langle\mathcal{C}_{1}\right\rangle \rightarrow \mathbb{K}\left\langle\mathcal{C}_{2}\right\rangle$. As $S$ is recognizable, there is a $\mathbb{K}$ - $\Sigma$-algebra $\mathscr{A}$ of finite rank and a $\mathbb{K}$ - $\Sigma$-epimorphism $\varphi: \mathbb{K}\left\langle\mathcal{C}_{2}\right\rangle \rightarrow \mathscr{A}$ such that $\operatorname{ker}(\varphi) \subseteq \operatorname{ker}(S)$. Hence, $\operatorname{ker}(\psi \circ \varphi) \subseteq \operatorname{ker}\left(\psi^{-1}(S)\right)$.

(Only if). Let $\Delta_{1} \subseteq \mathcal{C}_{1}$ be a finite generating set. Let $\psi^{-1}(S): \mathcal{C}_{1} \rightarrow \mathbb{K}$ be recognizable. Hence, $\mathscr{A}_{\psi^{-1}(S)}$ is of finite rank. We have

$$
\begin{aligned}
P_{1} \sim_{\psi^{-1}(S)} P_{2} & \Longleftrightarrow\left(\psi^{-1}(S), \tau\left[P_{1}\right]\right)=\left(\psi^{-1}(S), \tau\left[P_{2}\right]\right) \text { for all } \tau \in \operatorname{CTX}\left(\Sigma, \Delta_{1}\right) \\
& \Longleftrightarrow\left(S, \psi\left(\tau\left[P_{1}\right]\right)\right)=\left(S, \psi\left(\tau\left[P_{2}\right]\right)\right) \text { for all } \tau \in \operatorname{CTX}\left(\Sigma, \Delta_{1}\right) \\
& \Longleftrightarrow\left(S, \tau\left[\psi\left(P_{1}\right)\right]\right)=\left(S, \tau\left[\psi\left(P_{2}\right)\right]\right) \text { for all } \tau \in \operatorname{CTX}\left(\Sigma, \psi\left(\Delta_{1}\right)\right) \\
& \Longleftrightarrow \psi\left(P_{1}\right) \sim_{S} \psi\left(P_{2}\right) .
\end{aligned}
$$

There is, hence, an epimorphism from $\mathscr{A}_{\psi^{-1}(S)}$ to $\mathscr{A}_{S}$. Thus, we conclude that $\mathscr{A}_{S}$ is of finite rank, too.

Corollary 2.9. A series $S: \mathcal{C} \rightarrow \mathbb{K}$ is recognizable iff $\eta_{\mathcal{C}}^{-1}(S)$ is recognizable.

We now show that the proposed notion of recognizability coincides with the well-known notion of the behavior of weighted tree automata (over trees in $T_{\Sigma}(\Delta)$ ) (see e.g. [1]). A weighted tree automaton $\mathcal{A}$ is a tuple $(Q, \delta, \kappa)$ where $Q$ is a finite set of states, $\kappa: Q \rightarrow \mathbb{K}$ and $\delta=\left(\delta_{f}\right)_{f \in \Sigma \cup \Delta}$ is a family of mappings $\delta_{f}: Q^{\mathrm{rk}(f)} \rightarrow \mathbb{K}^{Q}$. We extend $\delta_{f}$ to $\delta_{f}: \underbrace{\mathbb{K}^{Q} \times \ldots \times \mathbb{K}^{Q}}_{\mathrm{rk}(f)} \rightarrow \mathbb{K}^{Q}$ by letting

$$
\delta_{f}\left(v_{1}, \ldots, v_{k}\right)_{q}=\sum_{q_{1}, \ldots, q_{k} \in Q} \delta_{f}\left(q_{1}, \ldots, q_{k}\right)_{q} \cdot\left(v_{1}\right)_{q_{1}} \cdot \ldots \cdot\left(v_{n}\right)_{q_{n}} .
$$

Note that the $\delta_{f}$ are multilinear. Hence, they turn $\mathbb{K}^{Q}$ into a $\mathbb{K}$ - $\Sigma$-algebra. Let $\delta: \mathbb{K}\left\langle T_{\Sigma}(\Delta)\right\rangle \rightarrow \mathbb{K}^{Q}$ be the $\mathbb{K}$ - $\Sigma$-homomorphism mentioned in Remark 2.3 extending $\delta: \Delta \rightarrow \mathbb{K}^{Q}: a \mapsto \delta_{a}$. Now, the behavior $\|\mathcal{A}\|: T_{\Sigma}(\Delta) \rightarrow \mathbb{K}$ of $\mathcal{A}$ is defined by $(\|\mathcal{A}\|, t)=\sum_{q \in Q} \delta(t)_{q} \cdot \kappa_{q}$. We say a formal tree series is regular if it is the behavior of a weighted tree automaton.

Proposition 2.10. Let $S: T_{\Sigma}(\Delta) \rightarrow \mathbb{K}$. Then $S$ is regular if it is recognizable.

Proof. Let $\mathscr{A}$ be of finite rank generated by $m_{1}, \ldots, m_{n}$ and $\varphi: \mathbb{K}\left\langle T_{\Sigma}(\Delta)\right\rangle \rightarrow \mathscr{A}$ a $\mathbb{K}$ - $\Sigma$-epimorphism such that $\operatorname{ker}(\varphi) \subseteq \operatorname{ker}(S)$. We set $Q=[n]:=\{1, \ldots n\}$. Let $f \in \Sigma \cup \Delta$ with $\operatorname{rk}(f)=k$ and let $i_{1}, \ldots i_{k} \in Q$. Then $\mu_{f}\left(m_{i_{1}}, \ldots, m_{i_{k}}\right)=$ $\sum_{1 \leq j \leq n} \delta_{f}\left(i_{1}, \ldots, i_{k}\right)_{j} m_{j}$ for some $\delta_{f}\left(i_{1}, \ldots, i_{k}\right)_{j} \in \mathbb{K}$. This defines $\delta_{f}: Q^{k} \rightarrow$ $\mathbb{K}^{Q}$. Since $\operatorname{ker}(\varphi) \subseteq \operatorname{ker}(S)$, there is a linear form $\gamma: \mathscr{A} \rightarrow \mathbb{K}$ such that $\gamma \circ \varphi=S$. We define $\kappa: Q \rightarrow \mathbb{K}$ by setting $\kappa(i)=\gamma\left(m_{i}\right)$ for all $1 \leq i \leq n$. Let $\mathcal{A}=(Q, \delta, \kappa)$. It is easy to see by induction that $\varphi(t)=\sum_{1 \leq j \leq n} \delta(t)_{j} m_{j}$. Hence, $\|\mathcal{A}\|=S$.

Similar to the proof of Reutenauer for Proposition 2.6 one shows for trees:

Proposition 2.11. Let $\mathbb{K}$ be a commutative ring or let $\mathbb{K}$ be a commutative and locally finite semiring. A tree series $S: T_{\Sigma}(\Delta) \rightarrow \mathbb{K}$ is recognizable iff it is regular. 
Remark 2.12. For the proofs of Propositions 2.6 and 2.11 one needs that finitely generated modules over finitely generated rings are Noetherian, i.e. any submodule is finitely generated. It is open whether the propositions hold for arbitrary commutative semirings.

\section{Relational Structures and Weighted Logics}

Let $\sigma=\left(\left(R_{i}\right)_{i \in I}, \rho\right)$ be a relational signature consisting of a family of relation symbols $R_{i}$ each of which is equipped with an arity through $\rho: I \rightarrow \mathbb{N}_{+}$. Let $s=\left(V(s),\left(R_{i}^{s}\right)_{i \in I}\right)$ be a $\sigma$-structure consisting of a domain $V(s)$ together with a relation $R_{i}^{s}$ of arity $\rho(i)$ for every relation symbol $R_{i}$. Subsequently, we assume that the domain is finite. Moreover, we will distinguish relational structures only up to isomorphisms. In the following, let $\mathcal{C}$ be a class of $\sigma$-structures.

We review classical MSO logic for relational structures over signature $\sigma=$ $\left(\left(R_{i}\right)_{i \in I}, \rho\right)$. Formulae of $\operatorname{MSO}(\sigma)$ are inductively built from the atomic formulae $x=y, R_{i}\left(x_{1} \ldots x_{\rho(i)}\right), x \in X$ using negation $\neg$, the connective $\vee$ and the quantifications $\exists x$. and $\exists X$. where $x, y, x_{j}$ are first-order variables and $X$ is a second-order variable.

Let $\varphi \in \operatorname{MSO}(\sigma)$ and let $\operatorname{Free}(\varphi)$ denote the set of variables that occur free in $\varphi$. Let $\mathcal{V}$ be a finite set of first-order and second-order variables. A $(\mathcal{V}, s)$ assignment $\gamma$ is a mapping from $\mathcal{V}$ to the power set $\mathscr{P}(V(s))$ such that first-order variables are mapped to singletons. For $v \in V(s)$ and $T \subseteq V(s)$ we denote by $\gamma[x \rightarrow v]$ and $\gamma[X \rightarrow T]$ the $(\mathcal{V} \cup\{x\}, s)$-assignment which equals $\gamma$ on $\mathcal{V} \backslash\{x\}$ (resp. $\mathcal{V} \backslash\{X\}$ ) and assumes $\{v\}$ for $x$ (resp. $T$ for $X$ ). Now, let $\operatorname{Free}(\varphi) \subseteq \mathcal{V}$ and $\gamma$ be a $(\mathcal{V}, s)$-assignment. We write $(s, \gamma) \models \varphi$ if $\varphi$ holds in $s$ under the assignment $\gamma$.

We write $\varphi\left(x_{1}, \ldots, x_{n}, X_{1}, \ldots, X_{m}\right)$ if $\operatorname{Free}(\varphi)=\left\{x_{1}, \ldots, x_{n}, X_{1}, \ldots, X_{m}\right\}$. In this case write $s \models \varphi\left[v_{1}, \ldots, v_{n}, T_{1}, \ldots, T_{m}\right]$ when we have $(s, \gamma) \models \varphi$ if $\gamma\left(x_{i}\right)=\left\{v_{i}\right\}$ and $\gamma\left(X_{i}\right)=T_{i}$. For $\varphi\left(x_{1}, \ldots, x_{k}\right) \in \operatorname{MSO}(\sigma)$ we define $\varphi^{s}=\left\{\left(v_{1}, \ldots, v_{k}\right) \in V(s)^{k} \mid s \models \varphi\left[v_{1}, \ldots, v_{k}\right]\right\}$. In the sequel, we identify the pair $(s, \gamma)$ with the relational structure which expands $s$ with additional unary relations $x^{s}=\gamma(x)$ and $X^{s}=\gamma(X)$ for each first-order variable $x \in \mathcal{V}$ and each second-order variable $X \in \mathcal{V}$. By $\sigma_{\mathcal{V}}$ we denote the corresponding signature and by $\mathrm{N}_{\mathcal{V}}$ the class of all $\sigma_{\mathcal{V}}$-structures $(s, \gamma)$ for $s \in \mathcal{C}$ and $\gamma$ a $(\mathcal{V}, s)$ assignment. Let $\varphi \in \operatorname{MSO}(\sigma)$ and $\mathcal{V} \supseteq \operatorname{Free}(\varphi)$ be a finite set of variables, then $\mathscr{L}_{\mathcal{V}}(\varphi)=\left\{(s, \gamma) \in \mathrm{N}_{\mathcal{V}} \mid(s, \gamma) \models \varphi\right\}$ and $\mathscr{L}(\varphi)=\mathscr{L}_{\text {Free }(\varphi)}(\varphi)$.

Let $Z \subseteq \operatorname{MSO}(\sigma)$. A language $L \subseteq \mathcal{C}$ is Z-definable if $L=\mathscr{L}(\varphi)$ for a sentence $\varphi \in Z$. $\operatorname{MSO}(\sigma)$-definable languages are simply called definable. Formulae containing no quantification at all are called propositional. First-order formulae, i. e. formulae containing only quantification over first-order variables are collected in $\mathrm{FO}(\sigma)$. The class $\operatorname{EMSO}(\sigma)$ consists of all formulae $\varphi$ of the form $\exists X_{1} \ldots \exists X_{m} . \psi$ where $\psi \in \mathrm{FO}(\sigma)$.

We now define weighted MSO logic as introduced in [5]. Formulae of $\operatorname{MSO}(\mathbb{K}, \sigma)$ are built from the atomic formulae $k$ (for $k \in \mathbb{K}$ ), $x=y$, $R_{i}\left(x_{1} \ldots x_{\rho(i)}\right), x \in X, \neg(x=y), \neg R_{i}\left(x_{1} \ldots x_{\rho(i)}\right), \neg(x \in X)$ using the con- 
nectives $\vee, \wedge$ and the quantifications $\exists x ., \exists X ., \forall x ., \forall X$.. Let $\varphi \in \operatorname{MSO}(\mathbb{K}, \sigma)$ and $\operatorname{Free}(\varphi) \subseteq \mathcal{V}$. The weighted semantics $\llbracket \varphi \rrbracket \mathcal{V}$ of $\varphi$ is a function which assigns to each pair $(s, \gamma) \in \mathrm{N}_{\mathcal{V}}$ an element of $\mathbb{K}$. For $k \in \mathbb{K}$ we put $\llbracket k \rrbracket \mathcal{V}(s, \gamma)=k$. For all other atomic formulae $\varphi$ semantics $\llbracket \varphi \rrbracket \mathcal{V}$ is given by the characteristic function $\mathbb{1}_{\mathscr{L}_{\mathcal{V}}(\varphi)}$. Moreover, we define

$$
\begin{array}{lll}
\llbracket \varphi \vee \psi \rrbracket \mathcal{V}(s, \gamma) & = & \llbracket \varphi \rrbracket_{\mathcal{V}}(s, \gamma)+\llbracket \psi \rrbracket \mathcal{V}(s, \gamma), \\
\llbracket \varphi \wedge \psi \rrbracket_{\mathcal{V}}(s, \gamma) & = & \llbracket \varphi \rrbracket_{\mathcal{V}}(s, \gamma) \cdot \llbracket \psi \rrbracket \mathcal{V}(s, \gamma), \\
\llbracket \exists x . \varphi \rrbracket \mathcal{V}(s, \gamma) & = & \sum_{v \in V(s)} \llbracket \varphi \rrbracket \mathcal{V} \cup\{x\} \\
\llbracket \exists X . \varphi \rrbracket \mathcal{V}(s, \gamma) & = & \sum_{T \subseteq V(s)} \llbracket \varphi \rrbracket \mathcal{V} \cup\{X\} \\
\llbracket \forall x . \varphi \rrbracket_{\mathcal{V}}(s, \gamma) & = & \prod_{v \in V(s)} \llbracket \varphi \rrbracket \mathcal{V} \cup\{x\}(s, \gamma[X \rightarrow v]), \\
\llbracket \forall X . \varphi \rrbracket \mathcal{V}(s, \gamma) & = & \left.\prod_{T \subseteq V(s)} \llbracket \varphi \rrbracket \mathcal{V} \cup\{X\}\right),
\end{array}
$$

We put $\llbracket \varphi \rrbracket=\llbracket \varphi \rrbracket_{\text {Free }(\varphi)}$. We give an example at the end of Section 5 ,

Remark 3.1. 1. A formula $\varphi \in \operatorname{MSO}(\mathbb{K}, \sigma)$ which does not contain a subformula $k \in \mathbb{K}$ can be interpreted as an unweighted formula.

2. Let $\mathbb{K}$ be the boolean semiring. Then it is easy to see that weighted logics and classical MSO logic coincide. In this case $k$ is either 0 (false) or 1 (true).

Lemma 3.2. Let $s$ be a $\sigma$-structure, $\varphi \in \operatorname{MSO}(\mathbb{K}, \sigma)$ and $\mathcal{V} \supseteq$ Free $(\varphi)$. Moreover, let $\gamma$ be a $(s, \mathcal{V})$-assignment. Then $\llbracket \varphi \rrbracket \mathcal{V}(s, \gamma)=\llbracket \varphi \rrbracket\left(s, \gamma_{\mid} \operatorname{Free}(\varphi)\right)$.

For words examples show that unrestricted application of universal quantification does not preserve recognizability. We follow Droste and Gastin [5] to resolve this.

Definition 3.3. A function $S: \mathcal{C} \rightarrow \mathbb{K}$ is a definable step function if $S=$ $\sum_{1 \leq j \leq m} k_{j} \mathbb{1}_{L_{j}}$ for $k_{j} \in \mathbb{K}$ and definable languages $L_{j} \subseteq \mathcal{C}$.

Lemma 3.4. Let $\varphi \in \operatorname{MSO}(\mathbb{K}, \sigma)$ and $\mathcal{V} \supseteq \operatorname{Free}(\varphi)$. Then $\llbracket \varphi \rrbracket$ is a definable step function iff $\llbracket \varphi \rrbracket \mathcal{V}$ is a definable step function.

Definition 3.5. A formula $\varphi \in \operatorname{MSO}(\mathbb{K}, \sigma)$ is restricted if it does not contain universal set quantification and whenever $\varphi$ has subformula $\forall x . \psi$, then $\llbracket \psi \rrbracket$ is a definable step function.

Let $Z \subseteq \operatorname{MSO}(\mathbb{K}, \sigma)$. A series $S: \mathcal{C} \rightarrow \mathbb{K}$ is $Z$-definable if $S=\llbracket \varphi \rrbracket$ for a sentence $\varphi \in Z$. $\operatorname{MSO}(\mathbb{K}, \sigma)$-definable series are simply called definable. Let $\operatorname{RMSO}(\mathbb{K}, \sigma)$ comprise all restricted formulae of $\operatorname{MSO}(\mathbb{K}, \sigma)$. Furthermore, let $\operatorname{REMSO}(\mathbb{K}, \Sigma)$ consist of all $\varphi \in \operatorname{RMSO}(\mathbb{K}, \sigma)$ having the form $\exists X_{1} \ldots \exists X_{m} . \psi$ with $\psi$ not containing any set quantification.

The following theorem extends the result of Droste and Gastin [5] to trees in $T_{\Sigma}(\Delta)$. The domain of a tree is a finite, nonempty, prefix-closed subset of $\mathbb{N}^{*}$ and it has relations for the node labeling and relations $\mathrm{E}_{i}(x, y)$ saying that $y$ is the $i$-th child of $x$. 
Theorem 3.6 (Droste \& Vogler [7]). Let $\mathbb{K}$ be a commutative semiring. A tree series $S: T_{\Sigma}(\Delta) \rightarrow \mathbb{K}$ is regular iff it is RMSO-definable iff it is REMSOdefinable.

We will show how to transfer this result to other relational structures using definable transductions. First, we need some preparing definitions.

Definition 3.7. Let $\varphi \in \operatorname{MSO}(\sigma)$.

1. We call $\varphi+$-disambiguatable (resp. +-RMSO-disambiguatable) if there is a formula (resp. restricted formula) $\varphi^{+}$such that $\llbracket \varphi^{+} \rrbracket=\mathbb{1}_{\mathscr{L}(\varphi)}$.

2. We call $\varphi$--disambiguatable (resp. --RMSO-disambiguatable) if there is a formula (resp. restricted formula) $\varphi^{-}$such that $\llbracket \varphi^{-} \rrbracket=\mathbb{1}_{\mathscr{L}(\neg \varphi)}$.

3. We call $\varphi$ disambiguatable (resp. RMSO-disambiguatable) if it is both +-disambiguatable and--disambiguatable (resp. +-RMSO-disambiguatable and--RMSO-disambiguatable).

For any +-disambiguatable (resp. +-RMSO-disambiguatable) formula $\varphi$ we choose an arbitrary but fixed formula (resp. restricted formula) $\varphi^{+}$such that $\llbracket \varphi^{+} \rrbracket=\mathbb{1}_{\mathscr{L}(\varphi)}$. We define $\varphi^{-}$analogously.

Remark 3.8. 1. Every propositional formula is RMSO-disambiguatable. Moreover, if $\mathbb{K}$ is idempotent, then any $\varphi$ is disambiguatable. If additionally $\varphi$ does not contain universal set quantification, then $\varphi$ is RMSO-disambiguatable.

2 . Let $\mathbb{K}=\mathbb{N}$. Consider the class of graphs without edges where the vertices are labeled with $a$ or $b$. Then $\forall x \cdot \operatorname{Lab}_{a}(x)$ is +-RMSO-disambiguatable but it is not--disambiguatable.

Using Theorem 3.6 and Doner's famous Büchi-type theorem for trees [4, we obtain

Lemma 3.9. Let $\mathcal{C}$ be the class of trees. Then every formula $\varphi \in \operatorname{MSO}(\sigma)$ is RMSO-disambiguatable.

The following lemma is a slight modification of Lemma 5.1 in [17.

Lemma 3.10 (Meinecke [17]). If there is a +-RMSO-disambiguatable formula $\varphi(x, y)$ such that $\left(\varphi^{+}\right)^{s}$ is a linear order for every $s \in \mathcal{C}$, then every firstorder formula is RMSO-disambiguatable.

\section{Definable Transductions}

In model theory it is common to interpret one relational structure in another. Courcelle [3] takes quite a constructive point of view by introducing the notion of definable transductions between classes of relational structures. There one derives a new structure by interpreting it in $m$ copies of a given structure. Here we only regard deterministic definable transductions which, therefore, we call definable functions. Let $\sigma_{1}$ and $\sigma_{2}=\left(\left(R_{i}\right)_{i \in I}, \rho\right)$ be two relational signatures and let $\mathcal{C}_{1}$ and $\mathcal{C}_{2}$ be classes of finite $\sigma_{1^{-}}$and $\sigma_{2}$-structures, respectively. 
Definition 4.1. $A\left(\sigma_{1}, \sigma_{2}\right)$-m-copying definition scheme (without parameter) is a tuple

$$
\mathcal{D}=\left(\vartheta,\left(\delta_{j}\right)_{1 \leq j \leq m},\left(\varphi_{l}\right)_{l \in I \star m}\right) \quad \text { where } I \star m=\left\{(i, \tilde{\jmath}) \mid i \in I, \tilde{\jmath} \in[m]^{\rho(i)}\right\}
$$

of formulae in $\operatorname{MSO}\left(\sigma_{1}\right)$ such that $\operatorname{Free}(\vartheta)=\emptyset$, Free $\left(\delta_{j}\right)=\left\{x_{1}\right\}$ and $\operatorname{Free}\left(\varphi_{l}\right)=$ $\left\{x_{1}, \ldots, x_{\rho(i)}\right\}$ for $l=(i, \tilde{\jmath}) \in I \star m$.

Let $\mathcal{D}$ be a $\left(\sigma_{1}, \sigma_{2}\right)$-m-copying definition scheme and let $s_{1} \in \mathcal{C}_{1}$ such that $s_{1} \models \vartheta$. Then define the $\sigma_{2}$-structure $\operatorname{def}_{\mathcal{D}}\left(s_{1}\right)=s_{2}=\left(V\left(s_{2}\right),\left(R_{i}^{s_{2}}\right)_{i \in I}\right)$ where $V\left(s_{2}\right)=$ $\bigcup_{1 \leq j \leq m} \delta_{j}^{s_{1}} \times\{j\}$ and $R_{i}^{s_{2}}=\left\{\left(v_{1}, j_{1}\right), \ldots,\left(v_{r}, j_{r}\right) \in V\left(s_{2}\right)^{r} \mid\left(v_{1}, \ldots, v_{r}\right) \in\right.$ $\left.\varphi_{i,\left(j_{1}, \ldots, j_{r}\right)}^{s_{1}}\right\}$ with $r=\rho(i)$. The function defined by $\mathcal{D}$ is given by $s_{1} \mapsto \operatorname{def}_{\mathcal{D}}\left(s_{1}\right)$.

Definition 4.2. A partial function $\Phi: \mathcal{C}_{1} \rightarrow \mathcal{C}_{2}$ is a definable function if there is a definition scheme $\mathcal{D}$ such that $\Phi=\mathbf{d e f}_{\mathcal{D}}$. If there is a $\mathcal{D}$ such that $\vartheta, \delta_{j}$ and $\varphi_{l}$ are disambiguatable, then $\Phi$ is an unambiguously definable function. If $\vartheta, \delta_{j}$ and $\varphi_{l}$ are RMSO-disambiguatable, then $\Phi$ is a RMSO-definable function.

Courcelle [3] showed that the preimage of a definable set under a definable function is again definable. We will show a similar result for series. Let $\Phi: \mathcal{C}_{1} \rightarrow$ $\mathcal{C}_{2}$ be a partial function with domain $\operatorname{dom}(\Phi)$ and let $S: \mathcal{C}_{2} \rightarrow \mathbb{K}$. Define $\Phi^{-1}(S)$ by letting $\left(\Phi^{-1}(S), s_{1}\right)=\left(S, \Phi\left(s_{1}\right)\right)$ for all $s_{1} \in \operatorname{dom}(\Phi)$ and $\left(\Phi^{-1}(S), s_{1}\right)=0$ otherwise.

Proposition 4.3. Let $\Phi: \mathcal{C}_{1} \rightarrow \mathcal{C}_{2}$ be a partial function.

1. Let $\Phi$ be unambiguously definable. If there is a +-disambiguatable formula $\varphi(x, y)$ such that $\left(\varphi^{+}\right)^{s_{1}}$ is a linear order for every $s_{1} \in \mathcal{C}_{1}$ and if $S: \mathcal{C}_{2} \rightarrow \mathbb{K}$ is definable, then so is $\Phi^{-1}(S)$.

2. Let $\Phi$ be RMSO-definable. If $S: \mathcal{C}_{2} \rightarrow \mathbb{K}$ is RMSO-definable, then so is $\Phi^{-1}(S)$.

Remark 4.4. To show Proposition 4.3 one translates formulae in $\operatorname{MSO}\left(\sigma_{2}\right)$ to formulae in $\operatorname{MSO}\left(\sigma_{1}\right)$ using an appropriate definition scheme $\mathcal{D}=\left(\vartheta,\left(\delta_{j}\right),\left(\varphi_{l}\right)\right)$. If $\vartheta^{+}, \delta_{j}^{+}, \delta_{j}^{-}, \varphi_{l}^{+}$and $\varphi_{l}^{-}$can be chosen in FO, then a translation can be given such that REMSO-definability is preserved.

\section{Definable and Recognizable Text Series}

A text is, roughly speaking, a word with an additional linear order. More precisely:

Definition 5.1. Let $\Delta$ be a finite alphabet. A text over $\Delta$ is a tuple $\left(V, \lambda, \leq_{1}, \leq_{2}\right)$ where $\leq_{1}$ and $\leq_{2}$ are linear orders over the domain $V$ and $\lambda: V \rightarrow \Delta$ is a labeling function. 
We consider texts as relational structures where the relations are given by the labeling and by $\leq_{1}$ and $\leq_{2}$. As usual, we identify isomorphic texts.

We now define an algebraic structure on texts following Hoogeboom and ten Pas [15]. A biorder is a pair of two linear orders, i. e. a text without labeling. Each biorder defines an operation - we obtain a new text by substituting given texts into the nodes of the biorder. These texts then become intervals of the new text in both the first and the second order. Subsets being intervals of both orders are called clans. A biorder is primitive if it has only trivial clans, i. e. the singletons and the domain itself.

Let $\Sigma$ be a finite set of primitive biorders of cardinality at least two and let $\operatorname{TXT}_{\Sigma}(\Delta)$ be the set of texts generated from $\Delta$ using $\Sigma$. Let txt $=\eta_{\operatorname{TXT}_{\Sigma}(\Delta)}$. Applying the theory of 2-structures developed by Ehrenfeucht and Rozenberg [9] one obtains that $\operatorname{TXT}_{\Sigma}(\Delta)$ is almost freely generated in the variety of all $\Sigma$ algebras from the singleton texts, i. e. from $\Delta$. Only the two biorders of cardinality two satisfy an associative law [15. Thus, different preimages of a text $\tau \in \operatorname{TXT}_{\Sigma}(\Delta)$ under txt only differ with respect to these two associativity laws. Let $\operatorname{sh}(\tau)$ be the preimage where the brackets are in the right most form. Clearly, $\operatorname{sh}^{-1}\left(\operatorname{txt}^{-1}(L)\right)=L$ for any $L \subseteq \operatorname{TXT}_{\Sigma}(\Delta)$. Hoogeboom and ten Pas call $\operatorname{sh}(\tau)$ the $r$-shape of $\tau$. They show

Theorem 5.2 (Hoogeboom \& ten Pas [15]). A language $L \subseteq \operatorname{TXT}_{\Sigma}(\Delta)$ is recognizable iff it is definable.

To prove it, they show that sh and txt are definable functions. Now, Lemma 3.9 implies:

Proposition 5.3. The natural epimorphism txt : $T_{\Sigma}(\Delta) \rightarrow \mathrm{TXT}_{\Sigma}(\Delta)$ is an RMSO-definable function.

Proposition 5.4. The function $\mathrm{sh}: \mathrm{TXT}_{\Sigma}(\Delta) \rightarrow T_{\Sigma}(\Delta)$ is RMSO-definable.

Proof (Sketch). Again we follow the idea in [15]. There a 2-copying scheme for sh is given. The formulae involved contain nested universal quantification over sets. The formula interpreting the label of an inner node of an r-shape in its text is e.g. in $\Sigma_{4}$. However, analyzing the formulae it turns out that any quantification only concerns clans. Hence, we can transform them into equivalent first-order formulae by identifying a clan with its first and its last element with respect to the first order, say. Now, any formula involved becomes a first-order formula. The result follows then from Lemma 3.10 .

Theorem 5.5. Let $\mathbb{K}$ be a commutative ring or let $\mathbb{K}$ be a commutative and locally finite semiring. Let $S: \operatorname{TXT}_{\Sigma}(\Delta) \rightarrow \mathbb{K}$ be a text series. Then the following are equivalent.

1. $S$ is recognizable.

2. $S$ is RMSO-definable.

3. $S$ is REMSO-definable. 
Proof (Sketch.). Let $S$ be recognizable. By Proposition 2.10 and Corollary 2.9 $\operatorname{txt}^{-1}(S)$ is regular. By Theorem $3.6 \mathrm{txt}^{-1}(S)$ is REMSO-definable. From the proofs of Proposition 5.4 and Lemma 3.10 in [17] we obtain a 2-copying definition scheme for sh consisting of restricted first-order formulae only. By Remark 4.4 $\mathrm{sh}^{-1}\left(\operatorname{txt}^{-1}(S)\right)=S$ is definable in REMSO and, hence, in RMSO.

It remains to show that $S$ is recognizable if it is RMSO-definable. Let $S$ be RMSO-definable. From Proposition 5.3 using Proposition 4.3 $(2), \operatorname{txt}^{-1}(S)$ is RMSO-definable and, hence, regular by Theorem 3.6. By Prop. 2.11 and Cor. 2.9 $S$ is recognizable.

Note that there is a +-disambiguatable formula $\varphi(x, y)$ such that $\varphi^{t}$ is the lexicographic order of positions for any $t \in T_{\Sigma}(\Delta)$. Using the result of Droste and Vogler on the coincidence of regular and definable tree series over commutative and locally finite semirings [7] and Proposition 4.3(1) we obtain the following theorem.

Theorem 5.6. Let $\mathbb{K}$ be a commutative and locally finite semiring. A text series $S: \operatorname{TXT}_{\Sigma}(\Delta) \rightarrow \mathbb{K}$ is definable iff it is recognizable.

A computable field is a field with computable operations $\left(+,-, \cdot,{ }^{-1}\right)$; e.g. the rationals.

Corollary 5.7. Let $\mathbb{K}$ be a computable field. It is decidable whether two given restricted sentences over texts $\varphi$ and $\psi$ satisfy $\llbracket \varphi \rrbracket=\llbracket \psi \rrbracket$.

Proof. The proof of Proposition 4.3 is effective and gives restricted tree formulae $\varphi^{\prime}$ and $\psi^{\prime}$ such that $\llbracket \varphi^{\prime} \rrbracket=\operatorname{txt}^{-1}(\llbracket \varphi \rrbracket)$ and $\llbracket \psi^{\prime} \rrbracket=\operatorname{txt}^{-1}(\llbracket \psi \rrbracket)$. Clearly, $\llbracket \varphi \rrbracket=\llbracket \psi \rrbracket$ iff $\llbracket \varphi^{\prime} \rrbracket=\llbracket \psi^{\prime} \rrbracket$. The latter can be decided by Corollary 5.9 of [7].

Similarly, using Corollary 6.7 of [7] we obtain

Corollary 5.8. Let $\mathbb{K}$ be a computable locally finite commutative semiring. It is decidable whether two given sentences over texts $\varphi$ and $\psi$ satisfy $\llbracket \varphi \rrbracket=\llbracket \psi \rrbracket$.

The following corollary sharpens one implication of Theorem 5.2 .

Corollary 5.9. A language $L \subseteq \operatorname{TXT}_{\Sigma}(\Delta)$ is definable iff it is definable in EMSO.

Example 5.10. Let $\mathbb{K}=\mathbb{Z}$ be the ring of integers. Let $\operatorname{Clan}\left(x_{1}, x_{2}\right)$ be a firstorder formula saying that for a text $\tau,\left\{x \in \tau \mid x_{1} \leq_{1} x \leq_{1} x_{2}\right\}$ is a proper clan. Consider

$$
\varphi=\exists x_{1}, x_{2} . \operatorname{Clan}\left(x_{1}, x_{2}\right)^{+} \wedge \forall x, y \cdot x_{1} \leq_{1} x, y \leq_{1} x_{2} \rightarrow\left(x \leq_{1} y \leftrightarrow y \leq_{2} x\right) .
$$

For a text $\tau,(\llbracket \varphi \rrbracket, \tau)$ gives the number of proper clans generated only from the biorder of cardinality two having two reversed orders. By Theorem $5.5 \llbracket \varphi \rrbracket$ is recognizable. 


\section{Alternating Texts and Weighted Parenthesizing Automata}

In this section let $\Sigma=\left\{o_{h}, \circ_{v}\right\}$ be the set of the two biorders of cardinality two, where for $\circ_{h}$ both orders coincide. Then $\operatorname{TXT}_{\Sigma}(\Delta)$, the set of the so-called alternating texts ( [10, p. 261]), is the free bisemigroup generated by $\Delta$; where a bisemigroup is a set together with two associative operations. Several authors have investigated the free bisemigroup as a fundamental, two-dimensional extension of classical automaton theory, see e.g. Ésik and Németh [12] and Hashiguchi et. al. (e.g. [13]). Ésik and Németh consider as a representation for the free bisemigroup the so-called sp-biposets. They define parenthesizing automata. Here we define weighted parenthesizing automata.

Definition 6.1. A weighted parenthesizing automaton (wpa for short) over $\Delta$ is a tuple $\mathcal{P}=\left(\mathcal{H}, \mathcal{V}, \Omega, \mu, \mu_{o p}, \mu_{c l}, \lambda, \gamma\right)$ where

1. $\mathcal{H}, \mathcal{V}$ are finite disjoint sets of horizontal and vertical states, respectively.

2. $\Omega$ is a finite set of parentheses, 1

3. $\mu:(\mathcal{H} \times \Delta \times \mathcal{H}) \cup(\mathcal{V} \times \Delta \times \mathcal{V}) \rightarrow \mathbb{K}$ is the transition function,

4. $\mu_{o p}, \mu_{c l}:(\mathcal{H} \times \Omega \times \mathcal{V}) \cup(\mathcal{V} \times \Omega \times \mathcal{H}) \rightarrow \mathbb{K}$ are the opening and closing parenthesizing functions and

5. $\lambda, \gamma: \mathcal{H} \cup \mathcal{V} \rightarrow \mathbb{K}$ are the initial and final weight functions.

A run $r$ of $\mathcal{P}$ is a certain word over the alphabet $(\mathcal{H} \cup \mathcal{V}) \times(\Delta \cup \Omega) \times(\mathcal{H} \cup \mathcal{V})$ defined inductively as follows. We also define its label lab $(r)$, its weight wgt $(r)$, its initial state $\operatorname{init}(r)$ and its final state $\operatorname{fin}(r)$.

1. $\left(q_{1}, a, q_{2}\right)$ is a run for all $\left(q_{1}, q_{2}\right) \in(\mathcal{H} \times \mathcal{H}) \cup(\mathcal{V} \times \mathcal{V})$ and $a \in \Delta$. We set

$$
\begin{aligned}
& \operatorname{lab}\left(\left(q_{1}, a, q_{2}\right)\right)=a \in \operatorname{TXT}_{\Sigma}(\Delta), \\
& \operatorname{init}\left(\left(q_{1}, a, q_{2}\right)\right)=q_{1} \text { and } \operatorname{fin}\left(\left(q_{1}, a, q_{2}\right)\right)=q_{2} .
\end{aligned}
$$

2. Let $r_{1}$ and $r_{2}$ be runs with $\operatorname{fin}\left(r_{1}\right)=\operatorname{init}\left(r_{2}\right) \in \mathcal{H}(\operatorname{resp} . \mathcal{V})$. Then $r=r_{1} r_{2}$ is a run having

$$
\begin{aligned}
& \operatorname{lab}(r)=\operatorname{lab}\left(r_{1}\right) \circ_{h} \operatorname{lab}\left(r_{2}\right) \quad\left(\operatorname{resp} . \operatorname{lab}(r)=\operatorname{lab}\left(r_{1}\right) \circ_{v} \operatorname{lab}\left(r_{2}\right)\right), \\
& \operatorname{wgt}(r)=\operatorname{wgt}\left(r_{1}\right) \cdot \operatorname{wgt}\left(r_{2}\right), \operatorname{init}(r)=\operatorname{init}\left(r_{1}\right) \text { and } \operatorname{fin}(r)=\operatorname{fin}\left(r_{2}\right) .
\end{aligned}
$$

3. Let $r$ be a run resulting from 2 such that $\operatorname{fin}(r)$, init $(r) \in \mathcal{H}$ (resp. $\mathcal{V})$. Let $q_{1}, q_{2} \in \mathcal{V}(\operatorname{resp} . \mathcal{H})$ and $s \in \Omega$. Then $r^{\prime}=\left(q_{1},\left({ }_{s}, \operatorname{init}(r)\right) r(\operatorname{fin}(r),)_{s}, q_{2}\right)$ is a run having

$$
\begin{aligned}
& \operatorname{lab}\left(r^{\prime}\right)=\operatorname{lab}(r), \operatorname{wgt}\left(r^{\prime}\right)=\mu_{\mathrm{op}}\left(\left(q_{1},(s, \operatorname{init}(r))\right) \cdot \operatorname{wgt}(r) \cdot \mu_{\mathrm{cl}}\left((\operatorname{fin}(r),)_{s}, q_{2}\right)\right), \\
& \operatorname{init}\left(r^{\prime}\right)=q_{1} \text { and } \operatorname{fin}\left(r^{\prime}\right)=q_{2} .
\end{aligned}
$$

${ }^{1}$ Contrary to the definition of Ésik and Németh we let $s \in \Omega$ represent both the opening and the closing parentheses. To help the intuition we write $(s \text { or })_{s}$. 
Let $\tau \in \operatorname{TXT}_{\Sigma}(\Delta)$. Since we do not allow repeated application of rule 3 , there are only finitely many runs with label $\tau$. If $r$ is a run with $\operatorname{lab}(r)=\tau, \operatorname{init}(r)=q_{1}$, fin $(r)=q_{2}$, we write $r: q_{1} \stackrel{\tau}{\rightarrow} q_{2}$. The behavior of $\mathcal{P}$ is a series $\|\mathcal{P}\|: \operatorname{TXT}_{\Sigma}(\Delta) \rightarrow$ $\mathbb{K}$ with

$$
(\|\mathcal{P}\|, \tau)=\sum_{q_{1}, q_{2} \in \mathcal{H} \cup \mathcal{V}} \lambda\left(q_{1}\right) \cdot \sum_{r: q_{1} \stackrel{\tau}{\rightarrow} q_{2}} \operatorname{wgt}(r) \cdot \gamma\left(q_{2}\right) .
$$

An alternating text series $S$ is regular if there is a wpa $\mathcal{P}$ such that $\|\mathcal{P}\|=S$.

Proposition 6.2. Let $S: \operatorname{TXT}_{\Sigma}(\Delta) \rightarrow \mathbb{K}$. Then $S$ is regular iff $\operatorname{txt}^{-1}(S)$ is regular.

From Theorem 3.6 and Propositions 5.3 and 5.4 we now conclude the following connection between weighted logics and weighted parenthesizing automata.

Theorem 6.3. Let $\mathbb{K}$ be any commutative semiring. An alternating text series is regular iff it is RMSO-definable iff it is REMSO-definable.

Corollary 6.4. Let $\mathbb{K}$ be a commutative ring or let $\mathbb{K}$ be a commutative and locally finite semiring. An alternating text series is regular iff it is recognizable.

Remark 6.5. The class of alternating texts is isomorphic to the class of spbiposets. There is an isomorphism that can be defined by propositional formulae (see e.g. [12]). Thus, the results of the last two sections hold as well for spbiposets.

Acknowledgments. The author thanks Pascal Weil, Manfred Droste and Dietrich Kuske for their helpful comments as well as an anonymous referee whose remarks resulted in improvements of the paper.

\section{References}

1. S. Bozapalidis. Equational elements in additive algebras. Theory of Computing Systems, 32(1):1-33, 1999.

2. S. Bozapalidis and A. Alexandrakis. Représentations matricielles des séries d'arbre reconnaissables. Theoretical Informatics and Applications, 23(4):449-459, 1989.

3. B. Courcelle. Monadic second-order definable graph transductions: a survey. Theoretical Computer Science, 126:53-75, 1994.

4. J. Doner. Tree acceptors and some of their applications. Journal of Computer and System Sciences, 4:406-451, 1970.

5. M. Droste and P. Gastin. Weighted automata and weighted logics. In Proc. of the 32nd ICALP, Lisbon, volume 3580 of Lecture Notes in Computer Science, pages 513-525, 2005.

6. M. Droste and G. Rahonis. Weighted automata and weighted logics on infinite words. In Proc. of the 10th DLT, Santa Barbara, volume 4036 of Lecture Notes in Computer Science, pages 49-58, 2006.

7. M. Droste and H. Vogler. Weighted tree automata and weighted logics. Theoretical Computer Science, 366:228-247, 2006. 
8. A. Ehrenfeucht, T. Harju, and G. Rozenberg. The Theory of 2-structures: A Framework for Decomposition and Transformation of Graphs. World Scientific, 1999.

9. A. Ehrenfeucht and G. Rozenberg. Theory of 2-structures. I and II. Theoretical Computer Science, 70:277-342, 1990.

10. A. Ehrenfeucht and G. Rozenberg. T-structures, T-functions, and texts. Theoretical Computer Science, 116:227-290, 1993.

11. A. Ehrenfeucht, P. ten Pas, and G. Rozenberg. Context-free text grammars. Acta Informatica, 31(2):161-206, 1994.

12. Z. Ésik and Z. L. Németh. Higher dimensional automata. Journal of Automata, Languages and Combinatorics, 9(1):3-29, 2004.

13. K. Hashiguchi, S. Ichihara, and S. Jimbo. Formal languages over free binoids. Journal of Automata, Languages and Combinatorics, 5(3):219-234, 2000.

14. H. J. Hoogeboom and P. ten Pas. Text languages in an algebraic framework. Fundamenta Informaticae, 25(3):353-380, 1996.

15. H.J. Hoogeboom and P. ten Pas. Monadic second-order definable text languages. Theory of Computing Systems, 30:335-354, 1997.

16. I. Mäurer. Weighted picture automata and weighted logics. In Proc. of the 23rd STACS, Marseille, volume 3884 of Lecture Notes in Computer Science, pages 313$324,2006$.

17. I. Meinecke. Weighted logics for traces. In Proc. of the 1st CSR, St. Petersburg, volume 3967 of Lecture Notes in Computer Science, pages 235-246, 2006.

18. J. Mezei and J. B. Wright. Algebraic automata and context-free sets. Information and Control, 11(1/2):3-29, 1967.

19. Ch. Reutenauer. Séries formelles et algèbres syntactiques. J. Algebra, 66:448-483, 1980. 